\title{
INTERMOLECULAR COMPLEX FORMATION BETWEEN METHYL ORANGE AND BOVINE SERUM ALBUMIN ON THE SURFACE OF HYDROXYAPATITE IN AN AQUEOUS PHASE
}

\author{
SABURO SHIMABAYASHI*, MAKIKO FUKUOKA, and TOMOAKI HINO \\ The Univ. of Tokushima, Fac. of Pharm. Sci., Div. of Phys. Pharm., \\ Sho-machi 1-78-1, Tokushima 770-8505, Japan \\ (E-mail address*: saburo@ph.tokushima-u.ac.jp)
}

\begin{abstract}
Methyl orange (MO) was not adsorbed directly to hydroxyapatite (HAP) when the concentration of MO was low, while bovine serum albumin (BSA) was adsorbed by HAP in the presence or absence of MO. Formation of the intermolecular complex between $\mathrm{MO}$ and BSA both in an aqueous phase and on the surface of HAP was discussed in the present paper. Apparent adsorption amount of MO to HAP increased with a concentration of BSA due to the adsorption of BSA and formation of the complex of MO-BSA on the surface when the [BSA] was low. The adsorption amount of MO, however, decreased with a concentration of BSA when the [BSA] was high. This fact was explained in terms of an increase in the concentration of BSA free from HAP, i.e., formation of the complex of MO-BSA in the mother solution.
\end{abstract}

\section{INTRODUCTION}

It was shown in the previous papers that hydroxyapatite $\left(\mathrm{Ca}_{10}\left(\mathrm{PO}_{4}\right)_{6}(\mathrm{OH})_{2}\right.$, HAP) adsorbs bovine serum albumin(BSA), which forms an intermolecular complex with another organic compound on the surface of HAP as well as in an aqueous phase. Sodium chondroitin-6-sulfate ( $\mathrm{NaChs}$ ) formed a surface complex with BSA at pHs lower than its isoelectric point (i.e.p. $=4.2-4.8)$ due mainly to electrostatic attractive force between them. ${ }^{1}$ The adsorption amount of BSA increased in the presence of a low concentration of sodium dodecylsulfate (SDS) by virtue of hydrophobic interaction between BSA and SDS adsorbed on the surface. The adsorption amount, however, decreased in the presence of a high concentration of SDS owing to the fact that the BSA molecule was expanded after its denaturation through the binding of SDS with BSA on

Received September 7, 2004; Accepted November 15, 2004 
the surface, resulting in an increase in its occupied area per molecule(unit in $\mathrm{m}^{2} /$ molecule) on the surface. ${ }^{2}$ Ponceau R (PR) also formed a complex with BSA on the surface. $^{3}$ An apparent adsorption amount of PR decreased after attaining a maximum with a concentration of BSA added to the system. This fact was explained as follows. The effect of formation of the complex of BSA-PR on the surface is dominant, i.e., PR was accumulated on the surface when the concentration of BSA was low. On the other hand, the effect of formation of the complex in the mother solution becomes dominant, i.e., an apparent desorption of PR from the surface to the mother solution was observed at a high concentration of BSA.

It was also shown in the previous papers that compounds having sulfate or sulfonate groups are easily adsorbed to HAP because their size and shape are quite similar to those of orthophosphate ion ( $\mathrm{PO}_{4}{ }^{3-}$ is a lattice and surface ion of HAP), resulting in isomorphous substitution ${ }^{4,5}$ between orthophosphate ion and the sulfate or sulfonate groups on the surface. ${ }^{3,6}$ Sodium dodecylsulfate, sodium dodecylsulfonate, and sodium dodecyl benzenesulfonate were easily adsorbed probably because these compounds have $-\mathrm{SO}_{4}{ }^{-}$or $-\mathrm{SO}_{3}{ }^{-}$terminal groups and hydrophobic groups which accelerate aggregation(i.e., ad- and hemimicelle formation) on the surface. ${ }^{6}$ On the other hand, PR has 2 sulfonate groups but its residue are less hydrophobic and more hydrophilic than these anionic surfactants. Driving force for the adsorption of PR, therefore, might be due mainly to high affinity of 2 sulfonate groups for HAP. In this context the affinity of MO for HAP should be studied, because it has just one sulfonate group in its molecular structure and the residue is rather hydrophilic similar to PR. We are expecting that there is difference in the adsorption affinity between $\mathrm{MO}$ and anionic surfactants mentioned above and also between MO and PR due to the number of sulfonate groups.

\section{EXPERIMENTAL}

Materials: BSA (MW: $6.6 \times 10^{4}$ ) and HAP (specific surface area: $50 \mathrm{~m}^{2} / \mathrm{g}$ ) used in this study were the same samples as that used in the previous paper. ${ }^{3}$ MO was a product of Nacalai Tesque, Inc.(Kyoto), of which chemical structure is shown in FIG.1. Other chemicals used in this study were purchased from Nacalai Tesque, Inc. or Wako Pure Chemicals Co. (Osaka), which were used without further purification. 


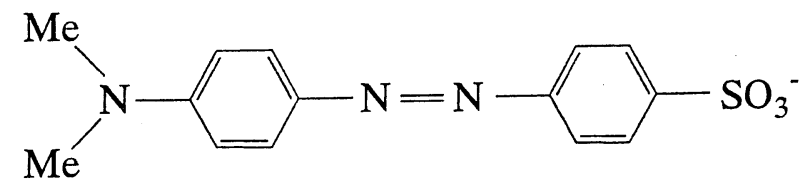

FUGURE 1. Chemical structure of methyl orange (MO)

Methods: Adsorption amounts of BSA and MO by HAP were obtained from the difference in their concentration before and after the adsorption by HAP $(=2.5 \mathrm{~g} / \mathrm{dl}$ at 5 or $30{ }^{\circ} \mathrm{C}$ ). The binding ratio of MO to BSA was determined through a dialysis method.

An equilibrium concentration of MO was determined by absorptiometry at 440 $\mathrm{nm}$, where is an isosbestic point of MO and BSA. Therefore, the MO concentration could be determined at this wave length irrespective of the concentration of BSA coexists. Typical relationship between the concentration of $\mathrm{MO}$ and absorption at 440 $\mathrm{nm}\left({ }^{440} \mathrm{~A}\right)$ is shown in FIG.2 (right). On the other hand, additivity was found in the absorption of BSA and that of MO at $279 \mathrm{~nm}$. BSA concentration, therefore, could be determined even in the presence of $\mathrm{MO}$, of which concentration was determined through the absorptiometry at $440 \mathrm{~nm}\left({ }^{440} \mathrm{~A}\right)$, mentioned above. The relationship between a concentration of BSA and absorption at $279 \mathrm{~nm}$ due to BSA $\left({ }^{279} \mathrm{~A}\right)$, which was obtained after correction for the absorption due to MO, is shown in FIG.2 (left). Solution pHs were 6.9-7.3 and 5.0-5.7 in the presence and absence of HAP. In this $\mathrm{pH}$ range or pHs higher than 4.4, exactly speaking, color of an aqueous solution of $\mathrm{MO}$ was orange yellow.

Surface tension of an aqueous solution was measured by means of a surface tensiometer (Du Nouy type, Shimadzu, Kyoto) at a room temperature $\left(=c a .28{ }^{\circ} \mathrm{C}\right.$ ). Anionic surfactants were dissolved in water. MO was dissolved in a phosphate buffer (pH 6.8) in order to keep the solution $\mathrm{pH}$ constant.

\section{RESULTS AND DISCUSSION}

\section{(1) Surface tension of an aqueous solution of sulfonated compounds}

Relationship between surface tension and the concentration is shown in FIG.3. Surface tension of an aqueous solution of an anionic surfactant, sodium dodecylsulfate and dodecyl sulfonate, steeply decreased with a concentration. On the other hand, that of MO gradually decreased. These results are showing that hydrophobicity of MO is 
weak because its residue is rather hydrophilic. It was shown in this meaning that $\mathrm{MO}$ is quite different from these surfactants in physicochemical properties of the adsorption.
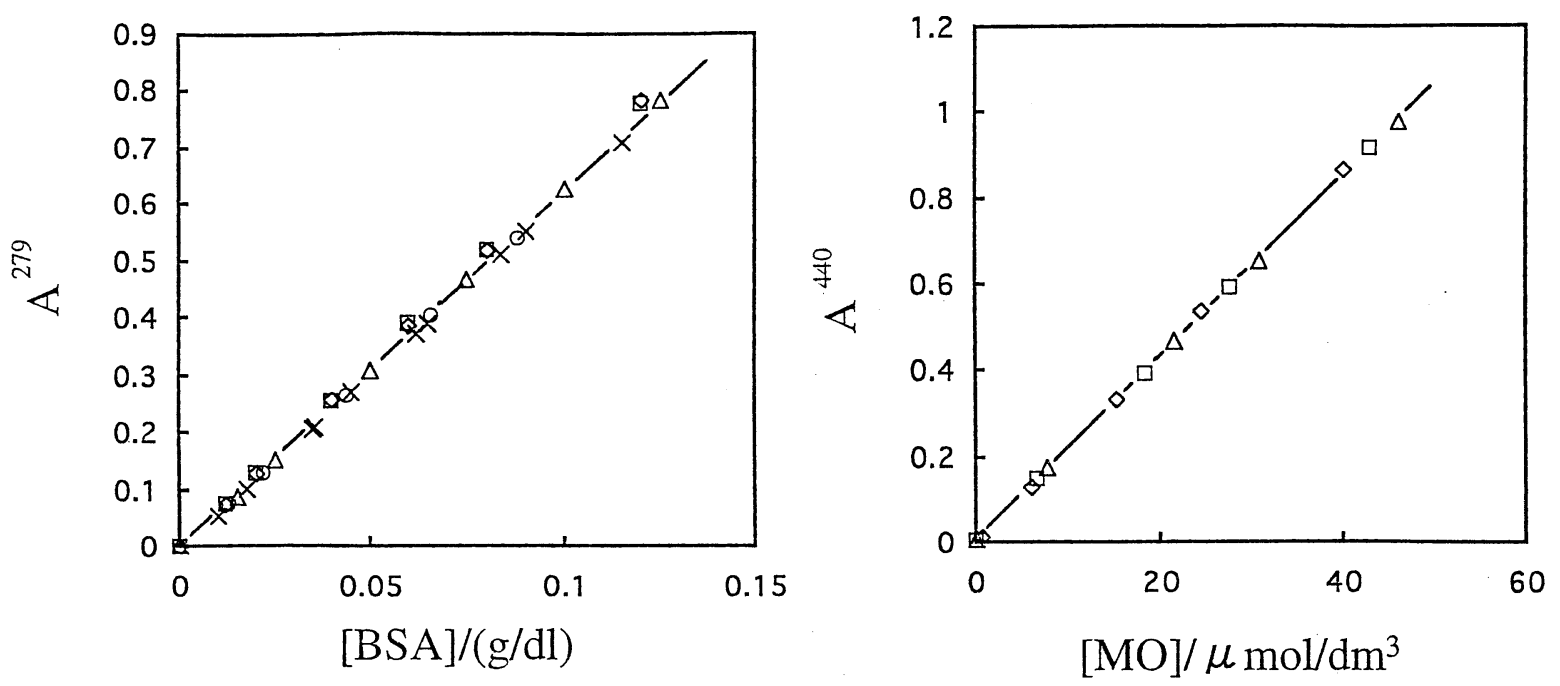

FIGURE 2. Relationship between optical absorption and concentration of a component in the presence of the other. (right) MO concentration vs. absorption at $440 \mathrm{~nm}$. [BSA] $/(\mathrm{g} / \mathrm{dl})=0$ (diamond), 0.08(square), 0.16(triangle). (left) BSA concentration $v s$. absorption at $279 \mathrm{~nm}$. [MO] $/\left(\mu \mathrm{mol} / \mathrm{dm}^{3}\right)=0$ (diamond), 7.54(square), 30.16(triangle), 48.25(circle), 60.32(cross).

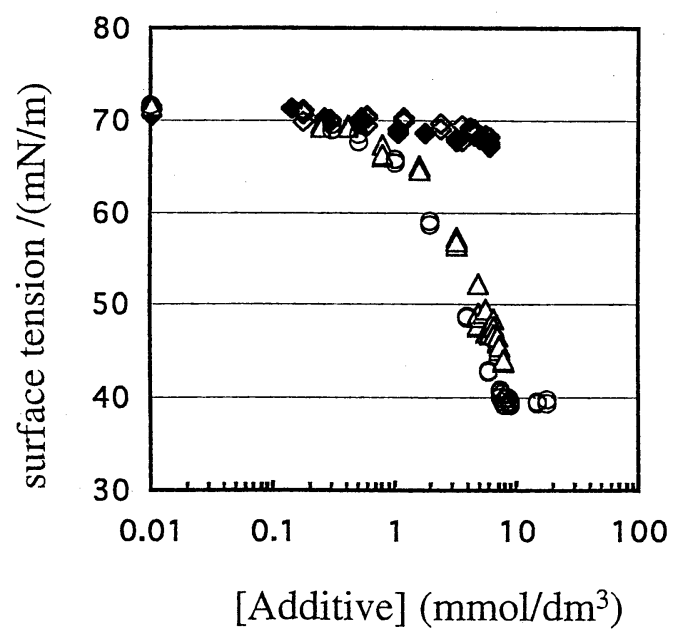

FIGURE 3. Surface tension as a function of a concentrarion of an aqueous solution of sodium dodecyl sulfonate(triangle), sodium dodecyl sulfate(circle). That for MO was obtained in a phosphate buffer at pH6.8 (open and closed diamond, two runs). 


\section{(2) Adsorption isotherms of MO and BSA by HAP}

MO was not adsorbed by HAP up to $0.3 \mathrm{mmol} / \mathrm{dm}^{3}$ in the absence of $\mathrm{NaCl}$ while up to $0.5 \mathrm{mmol} / \mathrm{dm}^{3}$ in the presence of $100 \mathrm{mmol} / \mathrm{dm}^{3} \mathrm{NaCl}$ (data not shown here). ${ }^{3}$ These results are showing that affinity of MO for HAP is rather weak, while the sulfated or sulfonated surfactants were adsorbed by virtue of aggregation effect of hydrophobic group on the surface. ${ }^{6}$ That is, formation of admicelle and hemimicelle on the surface is significant for the adsorption of these surfactants. ${ }^{7,8}$

BSA was adsorbed by HAP. FIG.4 shows a typical adsorption isotherm of BSA in the presence and absence of MO. The adsorption isotherm is of a high affinity type and there was little difference in the adsorption amount between in the presence and absence of MO. An initial slope of the adsorption isotherm of BSA became steeper with a concentration of added $\mathrm{NaCl}$, while a maximum adsorption amount (i.e., its saturated adsorption amount) was hardly changed with a concentration of added $\mathrm{NaCl}$ (data not shown here).

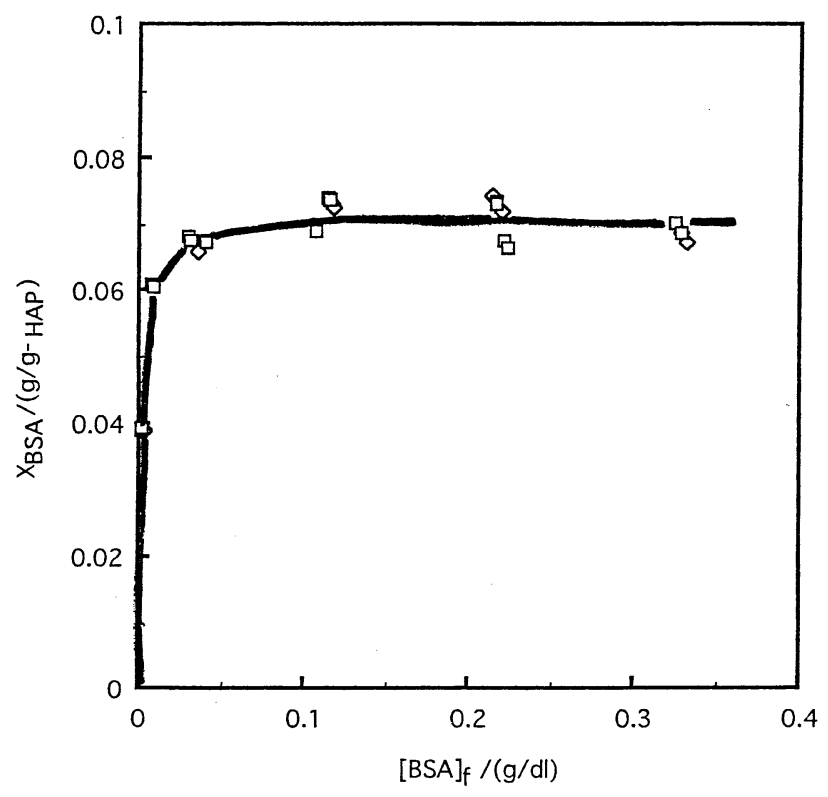

FIGURE 4. Adsorption isotherm of BSA on HAP in the presence or absence of MO. $[\mathrm{MO}] /\left(\mu \mathrm{mol} / \mathrm{dm}^{3}\right)=0$ (square) and 60 (diamond). $\mathrm{NaCl}$ was added by 100 $\mathrm{mmol} / \mathrm{dm}^{3}$ into a medium.

\section{(3) Binding of MO to BSA in the presence and absence of HAP}

Binding of MO to BSA was studied by means of a dialysis method in the presence and absence of HAP. HAP powder $(2.5 \mathrm{~g} / \mathrm{dl})$ was added in the compartment 
containing BSA, where BSA could capture MO molecules and also could be adsorbed to the surface of HAP, resulting in formation of the complex of BSA-MO on the surface of HAP as well as in the mother solution. The results are shown in FIG. 5.

The data in the absence of HAP are shown by open symbols while those in the presence of HAP by closed symbols. Both binding ratio increased with an equilibrium concentration of MO. The data shown are almost on a single curve regardless of the concentrations of HAP and BSA. This fact is showing that the binding ratio of MO to BAS was not affected whether BSA was adsorbed or not to HAP. This result is explained as follows. MO could be bound to BSA which is either adsorbed or free from HAP, while MO itself was not adsorbed directly to the surface of HAP, as mentioned above. BSA might not be denatuted after the adsorption and/or after the binding with MO. Therefore, its conformation and the number of the binding site for MO were not changed and the isotherm could be preserved even after the adsorption to HAP. The binding ratio decreased with a concentration of $\mathrm{NaCl}$ added (data not shown).

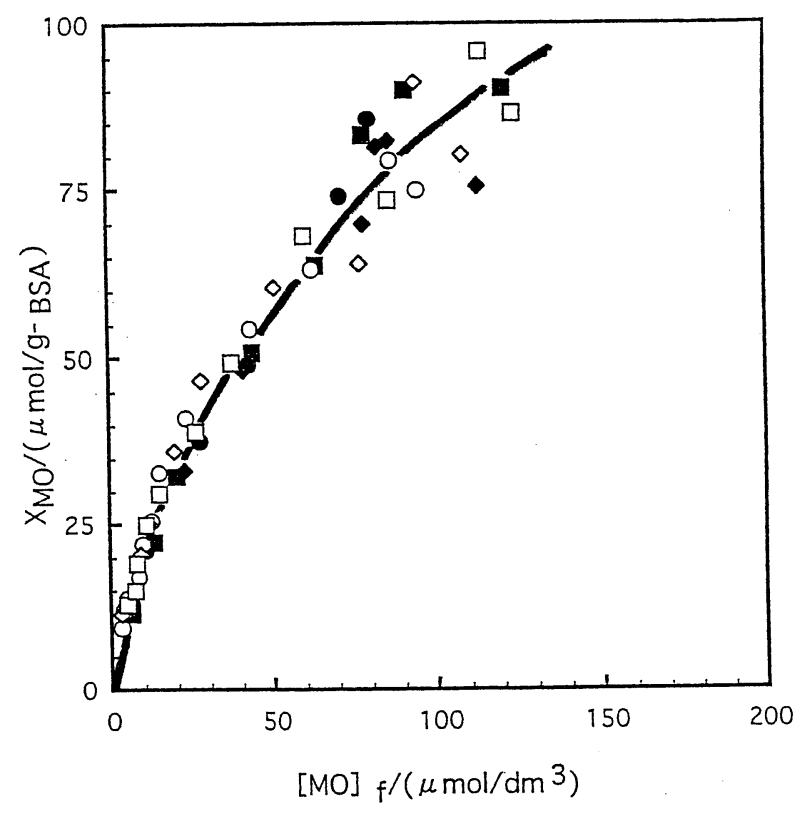

FIGURE 5. Binding isotherm of MO to BSA in the presence or absence HAP at $5{ }^{\circ} \mathrm{C} . \mathrm{NaCl}$ was not added here. Open symbols are showing the amount of binding in the absence of HAP. Concentration of BSA added, $[\mathrm{BSA}]_{\text {total }} /(\mathrm{g} / \mathrm{dl})=$ 0.075(open square), 0.10(open diamond), and 0.125(open circle). Closed symbols are showing that in the presence of $2.5 \mathrm{~g} / \mathrm{dl}$ HAP. Concentration of BSA added, $[\mathrm{BSA}]_{\mathrm{total}} /(\mathrm{g} / \mathrm{dl})=0.075$ (closed square), 0.10(closed diamond), and 0.125 (closed circle). 


\section{(4) Apparent adsorption amount of MO to HAP in the presence of BSA}

It may be said from another view point that $\mathrm{MO}$ originally unadsorbable to HAP became adsorbable by virtue of the adsorbed BSA which plays a roll of an intervener between HAP and MO. In order to more examine the effect of BSA, an apparent adsorption amount of MO to HAP, $\mathrm{X}_{\mathrm{MO}}$, was studied as a function of a concentration of BSA. The adsorption amounts were obtained by a one-pot batch method instead of a dialysis method used for FIG.5. The mixture of HAP, MO, and BSA in a beaker was filtered and/or centrifuged after attaining the adsorption equilibrium. The supernatant contains BSA and MO free from HAP, where some of the MO is free from BSA and the other is bound to BSA in an aqueous phase. The concentration of BSA in the presence of $\mathrm{MO},[\mathrm{BSA}]_{\mathrm{f}}$, and that of $\mathrm{MO}$ coexisting with BSA in the mother solution were determined by using the calibration curves shown in FIG.2. The amount of MO adsorbed by HAP in the presence of BSA, $\mathrm{X}_{\mathrm{MO}}$, was calculated. The relationship between $[\mathrm{BSA}]_{\mathrm{f}}$ and $\mathrm{X}_{\mathrm{MO}}$ are shown in FIG.6, where an initial concentration of $\mathrm{MO}$ was kept constant while that of BSA was changed.

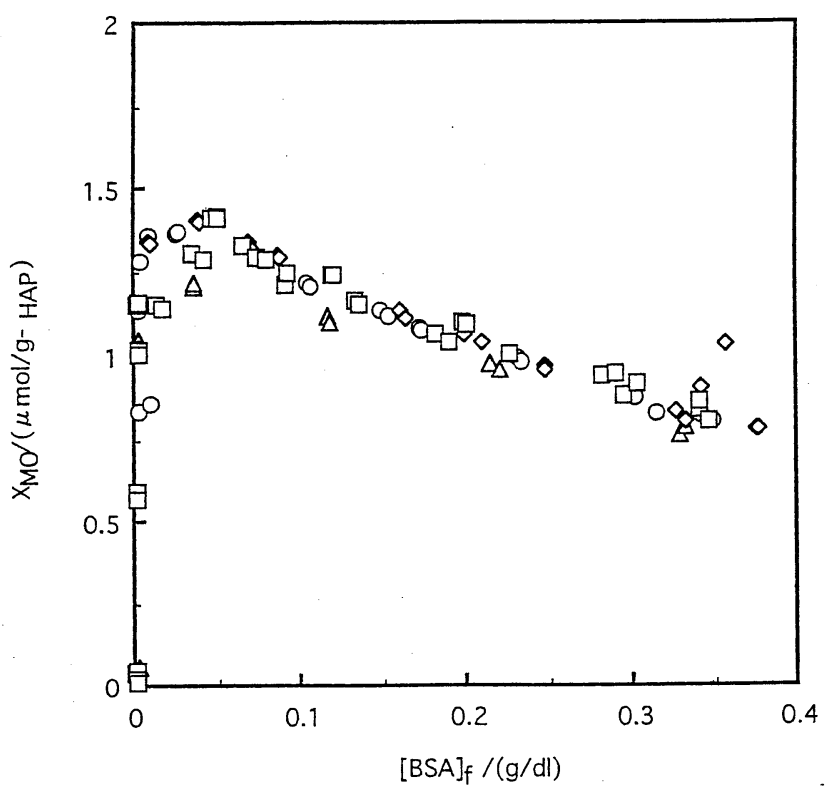

FIGURE 6. Apparent amount of MO adsorbed by HAP as a function of a concentration of BSA free from HAP, $[\mathrm{BSA}]_{\mathrm{f}}$, at $30{ }^{\circ} \mathrm{C}$. Concentrations of $\mathrm{MO}$ and HAP added were kept constant at $60 \mathrm{~mol} / \mathrm{dm}^{3}$ and $2.5 \mathrm{~g} / \mathrm{dl}$, respectively. Concentration of $\mathrm{NaCl}$ added $/\left(\mathrm{mmol} / \mathrm{dm}^{3}\right)=0$ (square), 30 (diamond), 50(circle), and 100(triangle). 
The amount of $\mathrm{X}_{\mathrm{MO}}$ steeply increased with $[\mathrm{BSA}]_{\mathrm{f}}$ when $[\mathrm{BSA}]_{\mathrm{f}}$ is low. In other words, the $\mathrm{X}_{\mathrm{MO}}$ increases with $\mathrm{X}_{\mathrm{BSA}}$, because $\mathrm{X}_{\mathrm{BSA}}$ also increases with $[\mathrm{BSA}]_{\mathrm{f}}$ (see FIG.2). However, the amount of $\mathrm{X}_{\mathrm{MO}}$ decreased after attaining a maximum at a high concentration of free BSA. This is due to the facts that $\mathrm{X}_{\mathrm{BSA}}$ is almost constant (i.e., close to the saturated amount), but $[\mathrm{BSA}]_{\mathrm{f}}$ increases (see FIG.2 also) and the BSA in the mother solution extracts the MO from the BSA-MO complex on the surface to form the complex in the mother solution.

FIGURE 7 shows the relationship between $\mathrm{X}_{\mathrm{MO}}$ and $[\mathrm{BSA}]_{\mathrm{f}} /[\mathrm{BSA}]_{\mathrm{b}}$, where $[\mathrm{BSA}]_{\mathrm{b}}$ shows the adsorption amount of BSA expressed in a concentration unit (i.e., concentration of added BSA $\left.-[\mathrm{BSA}]_{\mathrm{f}}\right)$. The amount of $\mathrm{X}_{\mathrm{MO}}$ decreased after attaining a maximum as the same manner as that shown in FIG.6 at each concentration of MO added to the system. The maximum point slightly moves to the right with an increase in a concentration of $\mathrm{MO}$ added.

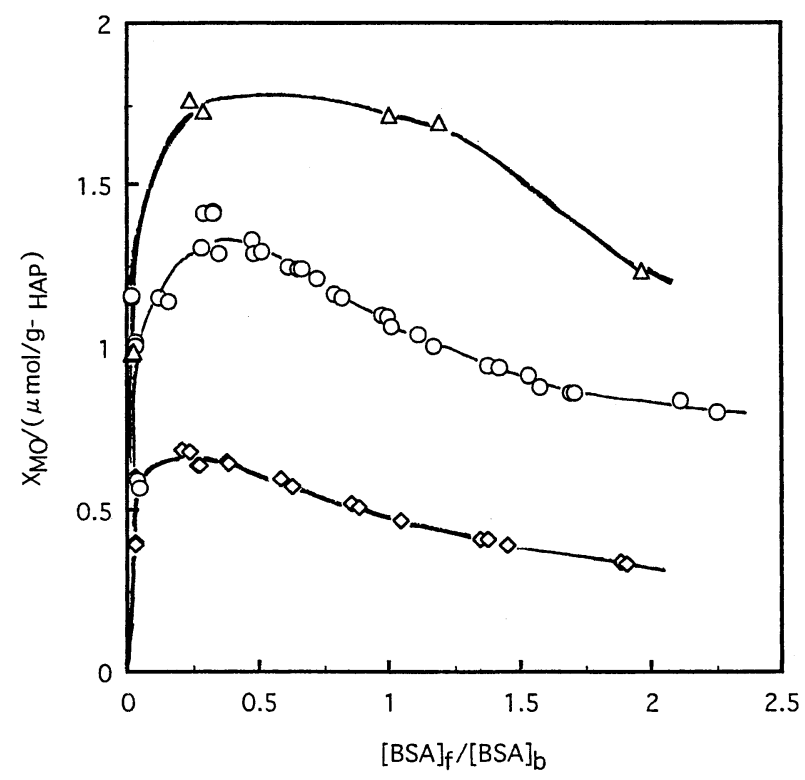

FIGURE 7. Apparent amount of MO adsorbed by HAP as a function of a concentration ratio of free $\mathrm{BSA}$ to bound $\mathrm{BSA},[\mathrm{BSA}]_{\mathrm{f}} /[\mathrm{BSA}]_{\mathrm{b}}$, at $30{ }^{\circ} \mathrm{C}$. Concentrations of $\mathrm{NaCl}$ and $\mathrm{HAP}$ added were kept constant at $0 \mathrm{~mol} / \mathrm{dm}^{3}$ and $2.5 \mathrm{~g} / \mathrm{dl}$, respectively. Concentration of $\mathrm{MO}$ added $/\left(\mathrm{mmol} / \mathrm{dm}^{3}\right)=30$ (diamond), 60 (circle), and 60(triangle).

\section{(5) Binding mechanism}

The binding scheme is shown in FIG.8. BSA and MO attain equilibrium of the 
binding both in an aqueous phase and on the surface. The driving force for the binding is mainly electrostatic attractive force between positive charge on BSA and negative charge on MO. Van der Waals force might be operating between them in some degree in the binding. Main driving force for the adsorption of BSA to HAP is electrostatic attractive force between positive charges on HAP $\left(\mathrm{Ca}^{2+}\right)$ and negative charge on BSA, and that between negative charges on HAP $\left(\mathrm{PO}_{4}{ }^{3-}\right)$ and positive charges on BSA. It is not clear in this study whether or not the complex of BSA-MO is adsorbed after the formation of it in an aqueous phase. MO was adsorbed by HAP in the presence of BSA by virtue of the complex formation. The complex formation was important for MO to be adsorbed. It was not adsorbed without the help of BSA. The decisive reason for it is not clear but some discussion is possible, as follows.

Sulfonated compounds such as anionic surfactants, mentioned above, were adsorbed. Cooperative interaction between hydrophobic tails on the surface (i.e., formation of admicelle and hemimicelle) ${ }^{6-7}$ as well as isomorphous substitution of sulfonate group with phosphate ion $^{4-8}$ was important for the adsorption. As for a water-soluble dye PR, it was adsorbed even though the residue is hydrophilic, probably because it has two sulfonate groups in its chemical structure and electrostatic attractive force toward positively charged site on HAP might be strong. ${ }^{3}$ As for MO, however, this compound has just one sulfonate group (FIG.1) and the residue is relatively hydrophilic (FIGS. 1 and 2). Therefore, the electrostatic attractive force toward HAP is weak and the surface aggregation on the HAP like a surfactant was practically impossible, resulting in low affinity of MO for HAP.
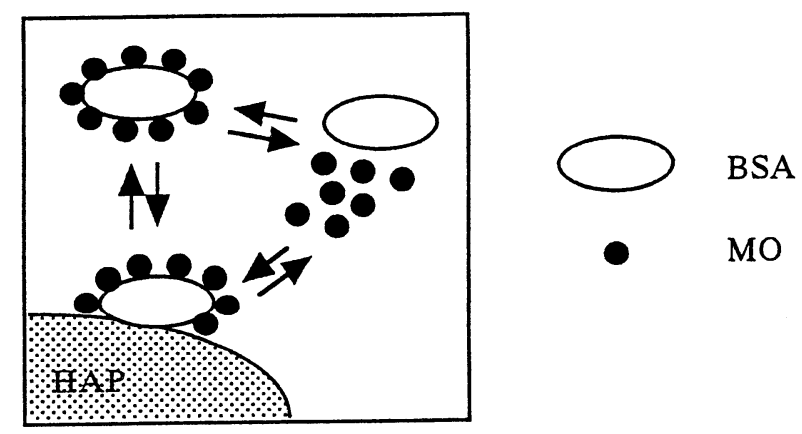

FIGURE 8. Scheme for the binding of MO, BSA, and HAP at the equilibrium. 


\section{SAMMARY}

1. The interactions between $\mathrm{MO}$ and BSA both in an aqueous phase and on the surface of HAP were studied. The binding ratio was almost the same between two phases.

2. The interaction between BSA and HAP was studied through the adsorption isotherms. BSA showed high affinity toward HAP. The adsorption amount was scarcely affected by the presence of $\mathrm{MO}$ and/or formation of the BSA-MO intermolecular complex.

3. The interaction between HAP and MO was studied. MO was hardly adsorbed by HAP in the absence of BSA while it was adsorbed in the presence of BSA, forming a surface complex of MO-BSA-HAP by intervening of the adsorbed BSA.

4. It was shown that not only sulfonate group but also hydrophobic group or more than two sulfonate groups is necessary for the compounds to be adsorbed by HAP. In the case MO, number of sulfonate groups in the molecule is one and the residue was hydrophilic. MO, therefore, was not adsorbed directly to the surface of HAP.

\section{REFERENCE}

1. S. Shimabayashi, K. Kishimoto, and T. Hino, Phosphorus Research Bulletin, 9, 23(1999).

2. S. Shimabayashi and M. Matsumoto, Phosphorus Research Bulletin, 2, 57(1992).

3. S. Shimabayashi, Y. Kato, M. Fukuoka, M. Hoshino, and T. Hino, Phosphorus Research Bulletin, 16, 55(2003).

4. S. Shimabayashi, Y. Tanizawa, K. Ishida, Chem. Pharm. Bull., 39, 2183(1991).

5. S. Shimabayashi, K. Furukawa, and T. Hino, in "Advances in Crystal Growth Inhibition Technologies", ed. by Z. Amjad, (Kluwer Academic/Plenum Publishers, New York, 2000), Chapter 4, pp.43-55.

6. S. Shimabayashi, M. Hoshino, and T. Hino, Phosphorus Research Bulletin, 14, 39(2002).

7. S. Shimabayashi, T. Hino, M. Hoshino, and M. Satomi, Phosphorus Research Bulletin, 12, 1(2001).

8. S. Shimabayashi, M. Kita, and T. Hino, Phosphorus Research Bulletin, 16, 47(2003). 